\title{
Descripción de factores no audiológicos asociados en adultos mayores del programa de audífonos año 2006, Hospital Padre Hurtado, Santiago
}

\section{Description of nonaudiologic associated factors in older adults from the 2006 hearing aid program at the Padre Hurtado Hospital in Santiago}

\author{
Oscar Cañete ${ }^{1}$, Ligia Gallardo $A^{2}$.
}

\begin{abstract}
RESUMEN
Introducción: En los últimos años se ha sugerido incorporar factores no audiológico en los programas de rehabilitación auditiva del adulto mayor.

Objetivo: Conocer factores sociales, familiares y de salud general de los adultos mayores (AM) beneficiarios del programa de audífonos año 2006 del Hospital Padre Hurtado, Santiago.

Material y método: Se entrevistaron pacientes cuya edad fue $\geq 65$ años atendidos en el Servicio de Otorrinolaringología durante el año 2006 que recibieron audífonos.

Resultados: Se realizó la entrevista al $87 \%$ de los beneficiados. El rango de edad de los pacientes fue de 65 a 98 años $(\bar{x}=77,6)$. Los resultados observados fueron los siguientes; a) en el $63 \%$ de los entrevistados el cuidador es un hijo (a), b) el $66 \%$ de los individuos presentaban limitaciones físicas (visuales o motrices, con o sin tratamiento) c) el $78 \%$ presenta algún tipo de ingreso económico (ejemplo pensión de vejez) sólo 2\% no presenta ingreso, d) el 7\% de los entrevistados son analfabetos, e) el $37 \%$ asiste a los controles médicos con su hijo (a), y f) sólo el 19\% participa en actividades sociales dentro de su comuna.

Conclusión: Los programas actuales de rehabilitación auditiva plantean la necesidad de considerar factores no audiológicos al momento de evaluar un paciente que requiere de algún tipo de ayuda técnica auditiva.
\end{abstract}

Palabras clave: Audífono, programa del adulto mayor (PAM), adulto mayor, audífonos en el adulto mayor, asistencia auditiva.

\begin{abstract}
Introduction: In recent years it has been suggested to include nonaudiologic factors in the rehabilitation programs for older adults.

Aim: The purpose of this study was to describe social, familial and general health factors affecting older adults (OA) beneficiaries of the 2006 hearing aid program at the Padre Hurtado Hospital in Santiago.
\end{abstract}

Tecnólogo Médico, Servicio de Otorrinolaringología, Hospital Padre Alberto Hurtado.

2 Asistente Social, Servicio de Atención al Paciente, Hospital Padre Alberto Hurtado. 
Material and Method: Patients, 65 year old or older, that received hearing aids at the ORL Department during 2006, were interviewed.

Results: $87 \%$ of patients that received a hearing aid were interviewed. Age range was 65 to 98 years old ( $x=77.6)$. Results can be summarized as follows: a) In $63 \%$ of the interviewed patients, the main caretaker was a son or daughter, b) $66 \%$ presented some kind of physical limitations (visual or motor, treated or non-treated), c) $78 \%$ had an income of some sort (pension, for example); only $2 \%$ had no income of any type, d) $7 \%$ of the interviewed individuals were illiterate, e) $37 \%$ attend medical controls accompanied by a son or daughter, and f) only $19 \%$ assists regularly to social activities within their neighborhood.

Conclusions: Current auditory rehabilitation programs pose the need of considering nonaudiologic factors when evaluating a patient that requires some type of hearing aid.

Key words: Hearing aid, older adult program, older adult, hearing aid in older adults, auditory assistance.

\section{INTRODUCCIÓN}

La presencia de una pérdida auditiva en la población general aumenta a medida que se avanza en edad, es así como ésta se encuentra entre $25 \%$ y $40 \%$ en personas sobre los 65 años, $50 \%$ en aquellos sobre 75 años llegando a $80 \%$ en personas sobre los 85 años ${ }^{1,2}$. Según datos de la encuesta nacional de salud (2003) existe una disminución de la agudeza auditiva que va entre $75,9 \%$ y $83,5 \%$ en la población mayor de 65 años ${ }^{3}$. De todas las modalidades sensoriales la audición es esencial en la comunicación; una pérdida auditiva afecta en su globalidad la calidad de vida de una persona alterando los procesos mentales, emocionales, educacionales y ocupacionales de un individuo. En el caso del AM esto puede traer consigo aislamiento, depresión, desinterés de las actividades diarias así como frustración con los miembros de la familia y amigos ${ }^{4}$.

En el año 1996 las personas mayores de 60 años constituían el 9,8\% de la población chilena, para el año 2002 esta cifra creció a 11,4\% ${ }^{5}$. Este aumento de la población de adultos mayores en Chile hace necesario la planificación, ejecución y control de programas de asistencia que permitan brindarles la atención necesaria. Dentro de los programas de rehabilitación auditiva (RA) los sistemas de asistencia auditiva, como los audífonos, son el pilar fundamental ${ }^{6,7}$, siendo éstos efectivos para revertir el impacto negativo social, emocional y comunicacional causado por una pérdida auditiva ${ }^{8}$.

Durante los últimos años se ha sugerido considerar factores no audiológicos en la evaluación de los pacientes que ingresan a estos programas de RA. Por ejemplo, la presencia de una deficiencia visual no tratada puede afectar negativamente el óptimo desempeño auditivo del paciente usuario de audífono $0^{9-11}$. Identificar la presencia de estos factores sociales, familiares y de salud permitiría lograr un enfoque biosicosocial con énfasis en la recuperación de las habilidades funcionales globales del individuo más que en sólo una función en particular como lo es la audición.

El presente estudio tuvo como objetivo describir los factores, sociales, familiares y de salud general observados en los adultos mayores (AM) beneficiarios del programa de audífonos año 2006 del Hospital Padre Hurtado, Santiago.

\section{MATERIAL Y MÉTODO}

Se entrevistaron 70 pacientes $(n=80)$ atendidos en el Servicio de Otorrinolaringología del Hospital Padre Hurtado de Santiago que presentaban indicación de uso de audífono. Todos fueron beneficiarios del programa del adulto mayor del año 2006. Los pacientes incluidos fueron estudiados con audiometría y prueba de audífonos, procedimientos solicitados por el médico tratante. Cada pacien- 
te fue implementado posteriormente en forma monoarual con un audífono digital retroauricular. Se excluyeron 10 pacientes los cuales no fueron entrevistados por inasistencia, déficit cognitivo importante, fallecimiento.

La encuesta utilizada se estructuró en 3 áreas de interés: la primera se refiere a conocer quién es el encargado de acompañar al AM a los controles médicos así como quién es el cuidador de éste, una segunda referida al tipo de ingreso económico y limitaciones (físicas, cognitivas, visuales) y una tercera referida al nivel de alfabetismo y participación en actividades sociales. Esta encuesta se realizó en formato de entrevista por el profesional a cargo del programa. Anexo $\mathrm{N}^{0} 1$.

La encuesta se realizó dentro del marco del programa del adulto mayor de entrega de audífonos con la finalidad de estructurar un programa de rehabilitación auditiva en esta población. Toda la información recolectada quedó registrada en la ficha clínica correspondiente siendo su uso exclusivo de este programa. Al momento de realizar la entrevista el profesional a cargo solicitó la autorización correspondiente. Para el analisis esta- dístico de los datos se utilizaron medidas de resumen (descriptivas).

\section{RESULTADOS}

Se evaluaron 70 pacientes lo que correspondió al $87 \%$ de los individuos beneficiados en el programa durante el año 2006. El rango de edad de los pacientes evaluados fue entre 65 y 98 años, cuyo promedio de edad fue de 77,6 años. El $45 \%$ correspondió a pacientes de sexo femenino y $55 \%$ a sexo masculino.

Al indagar sobre a) ¿Quién lo (a) acompaña a sus controles médicos? Se puede observar que $34 \%$ de los pacientes asiste solo a sus controles (Figura 1). b) ¿Quién es el cuidador del adulto mayor?, en $63 \%$ el hijo es el cuidador del AM (Figura 2). c) ¿Qué limitaciones físicas o cognitivas presenta?; cerca de $50 \%$ presenta una limitación visual (Figura 3). d) ¿Qué tipo de ingreso presenta?; más del $90 \%$ presenta algún tipo de ingreso económico (Figura 4). e) Al consignar nivel de alfabetismo se pudo observar que sólo $7 \%$ se

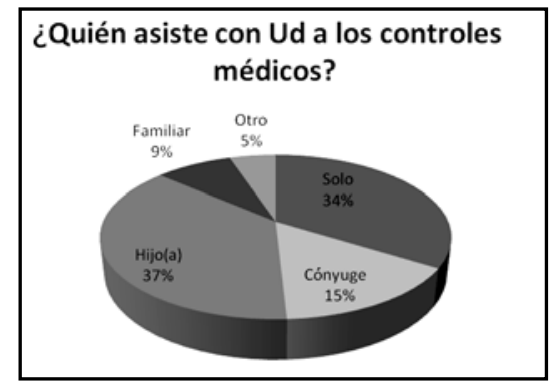

Figura 1.

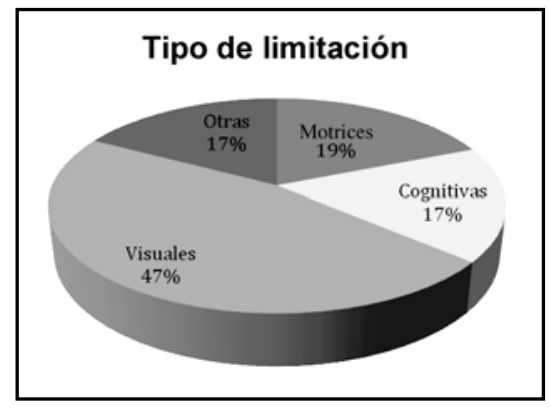

Figura 3.

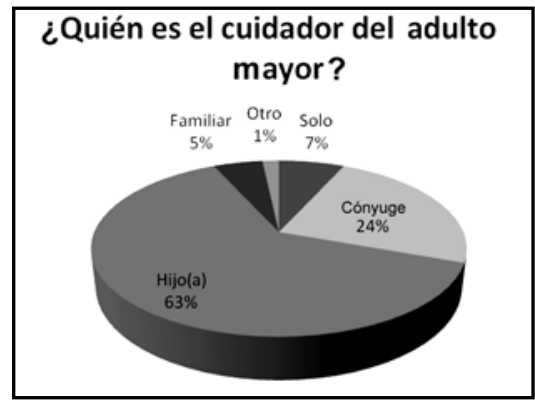

Figura 2

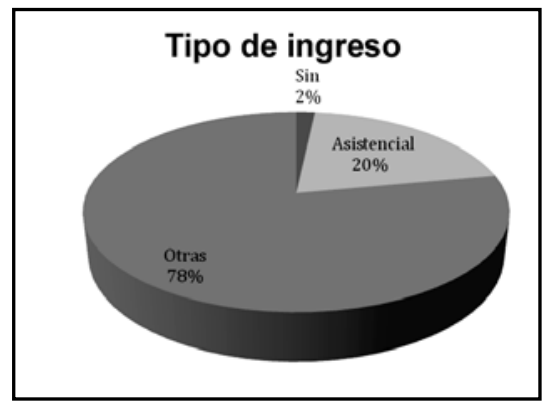

Figura 4. 


\section{ANEXO №1}

Ministerio de Salud

Gerencia de Calidad

Servicio de Atención al Paciente

Servicio Social /LGA

Descripción del Beneficiario

Nombre:

RUT:

Estado civil

Dirección:

Comuna:

Teléfono:

Consultorio de atención primaria:

Edad:

Presenta algún tipo de limitación:
$\square$ Visual
Cognitiva (concentración, memoria, etc)
Física

El paciente es:

$\square$ Analfabeto

Lee y escribe con dificultad

Lee y escribe sin dificultad

Red de Apoyo

- Paciente viene acompañado por (indicar nombre y relación con el paciente):

- Paciente es cuidado por (indicar nombre y relación con el paciente):

- El cuidador ¿vive con el paciente?: $\square$ Si $\square$ No

Observaciones (anotar si vive con alguien más):

- ¿El cuidador también es Adulto Mayor?: $\square \mathrm{Si} \quad \square$ No

- ¿Paciente mantiene participación o pertenencia en grupos comunitarios?

$\square \mathrm{Si}$, ¿Cuáles?

No, ¿por qué?

\section{Situación Socioeconómica}

- Los ingresos del paciente provienen de:

$\square$ PASIS $\square$ Compañía de seguro
$\square$ INP $\quad \square$ Sin pensión
$\square$ Vejez $\square$ Invalidez
$\square$ AFP $\quad \square$ Otro (mencionar)

- Constatar otros problemas sociales importantes existentes en el hogar del adulto mayor (principalmente uso de alcohol, drogas, violencia, de algún miembro de familia 0 del mismo paciente):

Nombre del profesional que entrevistó:

Fecha:

Se sugiere visita domiciliaria por asistente social:

Si $\square$ No 
declara analfabeto (Figura 5). f) ¿Participa en actividades sociales?, sólo 19\% participa en actividades sociales (Figura 6).

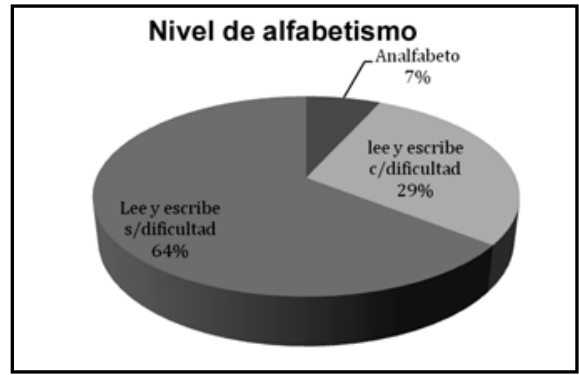

Figura 5.

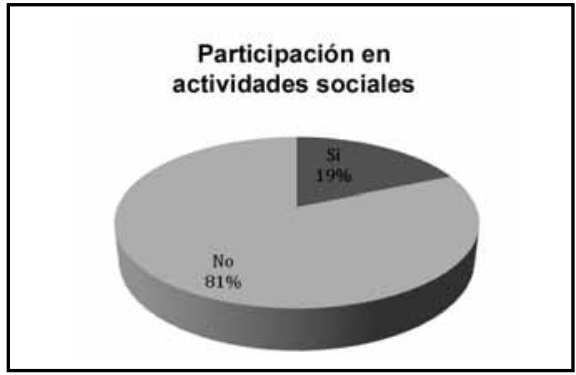

Figura 6.

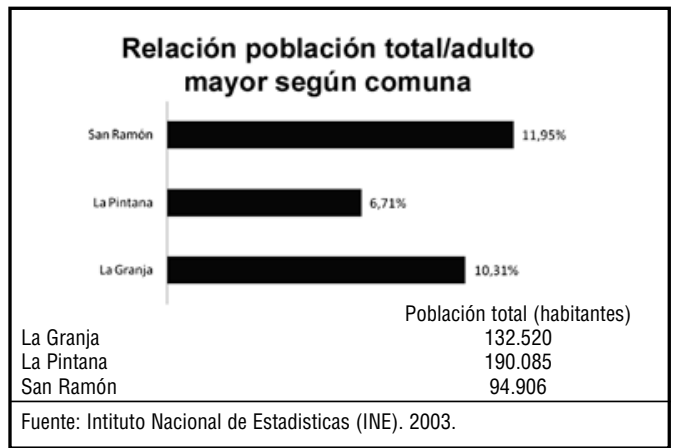

Figura 7.

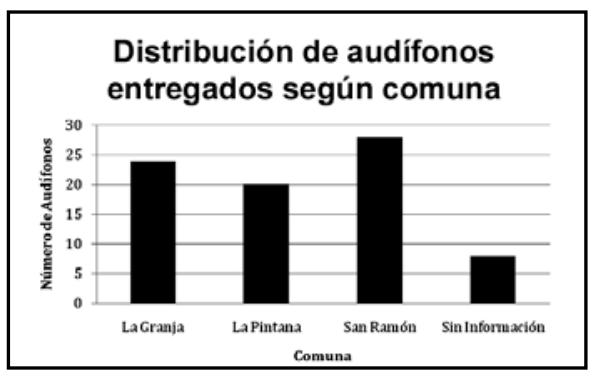

Figura 8.
A modo de información complementaria se presenta el porcentaje de AM según comunas atendidas por el hospital (Figura 7). Asímismo se presenta la distribución de los audífonos entregados según comuna de residencia del beneficiario, siendo ésta de 35\% para la comuna de San Ramón, 30\% para La Granja, $25 \%$ para la Pintana y $10 \%$ no se contaba con información (Figura 8).

\section{DISCUSIÓN}

El objetivo de aplicar una entrevista a los pacientes AM que son incluidos en un programa de RA es conocer algunas características sociales y familiares de estos pacientes permitiendo así considerar antecedentes que permitan intervenir en el entorno del AM, minimizando factores de riesgo, que impiden el uso óptimo del aparato entregado. De esta forma se complementa la información clínica con una visión integral del AM incorporando preguntas que buscan identificar factores protectores y de riesgo en él y su entorno.

La identificación de la red de apoyo con la que cuenta el AM usuario de audífono es una variable que no es considerada en los programas de asistencia auditiva en Chile, pero que es un factor de importancia en este proceso $0^{12}$. Con esta finalidad se indaga sobre: quién acompaña al AM a la entrevista (y controles médicos), quién cuida al AM, si ese cuidador vive con el AM, si el cuidador también es AM, y por último si el AM entrevistado participa en alguna instancia comunitaria (social).

Conocer la existencia de un cuidador del paciente nos permite dejar en evidencia la carencia de una red de apoyo, y en caso de existir un cuidador bien definido, sondear su grado de compromiso con los AM cuidados. Es así como, por ejemplo, al cruzar la información de un paciente que identifica a su hija como cuidadora, pero que al mismo tiempo asiste solo a sus controles, esta información nos dará luces de que eventualmente el individuo identificado como cuidador en la práctica no tiene las posibilidades de realizar esta función a cabalidad. Este punto es importante ya que en muchas oportunidades el apoyo que el AM puede recibir es de vital importancia en el buen uso, cuidado y beneficios entregados por el aparato de asistencia ${ }^{12}$.

Al momento de indagar sobre ¿quién acompaña al AM a los controles? y ¿quién es el cuidador?, 
respectivamente llama la atención que sólo $5 \%$ de los entrevistados define que no tiene cuidador, sin embargo $34 \%$ de los pacientes asisten solos a los controles. Lo que resulta más revelador es que este adulto mayor con "dificultades auditivas" y muy probablemente con alguna otra dificultad que limita su autovalencia (visuales, cognitivas, motrices y analfabetismo), asista solo a los controles médicos, y a recibir instrucciones que si no están adecuadas a su condición, no serán comprendidas por éste. Siguiendo con el análisis de estos resultados se puede inferir que muchos AM son cuidados también por otros adultos mayores ya que $24 \%$ reconoce como cuidador a su cónyuge, que en su mayoría son personas de edades similares a las del beneficiario.

En términos generales se ha podido observar que si no existe un apoyo continuo y global del paciente al cual se le implementa con algún sistema de asistencia auditiva (audífonos) la tasa de uso de estos aparatos es baja ${ }^{13}$.

Existe una alta prevalencia de una pérdida auditiva asociada a otras limitaciones funcionales en los adultos mayores ${ }^{14,15}$. Se han descrito factores físicos del paciente que pueden afectar un proceso de rehabilitación auditiva en los AM; dentro de los más importantes están las limitaciones visuales, motrices y $\operatorname{cognitivas~}^{12}$. La identificación de estas limitaciones es de suma importancia: problemas visuales sin tratamiento pueden afectar la discriminación auditiva disminuyendo por lo tanto su competencia comunicativa, problemas motrices comprometen el correcto manejo y postura del audífono ${ }^{12,16,17}$. Estudios han demostrado que alteraciones motrices pueden influir en el uso 0 no de un audífono en la población de $\mathrm{AM}^{18}$. Las limitaciones cognitivas juegan también un papel no menos preponderante en el proceso de rehabilitación auditiva ${ }^{19}$; un deterioro cognitivo importante puede afectar el éxito del proceso de amplificación. Cuando nuestro esfuerzo se enfoca sólo en la identificación de lo escuchado, el proceso mental de "almacenar" la información y construir un significado se ve afectado en forma importante. Sin embargo se ha podido observar que cualquier esfuerzo para mejorar el procesamiento auditivo (por ejemplo mejorando la audibilidad) logra que esta identificación sea menos dificultosa favoreciendo a otras funciones de orden superior como la memoria de trabajo ${ }^{19}$.
Al momento de conocer cuáles son las limitaciones que coexisten junto con la hipoacusia en los AM estudiados se le indicó que señalaran la presencia de algún tipo de limitación sin importar el tipo de ésta (ejemplo dificultad para leer, para ver, para caminar, uso de bastones, etc). De lo señalado pudimos observar que las limitaciones visuales $(47 \%)$ son las que se presentan con mayor frecuencia, seguido por las limitaciones motrices $(19 \%)$ y las cognitivas (17\%). Aspectos prácticos como verificar el buen estado de los lentes ópticos, derivación al especialista 0 el agrandar la tipografía de cualquier material por escrito que se le entregue al adulto mayor, surgen como solución para atender a nuestros beneficiarios de manera más integral. El detectar graves dificultades motrices (como mal de Parkinson por ejemplo) lleva nuestra preocupación al ámbito del cómo será la manipulación del aparato entregado.

Estas limitaciones deben ser evaluadas y tratadas por los profesionales correspondientes no sólo con la finalidad de lograr un rendimiento comunicativo efectivo sino que también pensando el mejoramiento de la calidad de vida del AM que las presenta.

Respecto a los ingresos económicos del AM, éste es un dato que nos habla de capacidad de ser independiente económicamente de su familia y así como los otros datos solicitados, también éste tiene un componente de análisis y otro práctico, porque se puede orientar al AM que no posee pensión para que pueda optar por alguna adecuada a sus características.

De los AM evaluados pudimos observar que un mayor porcentaje $(79 \%)$ tiene algún tipo de pensión entregada por alguna de las siguientes instituciones: Instituto de Normalización Previsional (INP), Administradora de Fondos de Pensiones (AFP), compañía de seguros y un porcentaje menor (21\%) cuenta con una Pensión Asistencial aportada por el Estado a través de los municipios, sólo $2 \%$ de los pacientes no presenta ningún tipo de ingreso. El monto de la pensión asistencial es considerablemente menor a otro tipo de pensiones. De los datos recolectados cerca de $80 \%$ de los AM evaluados presenta algún tipo de ingreso económico, es importante señalar que las comunas de residencia de los AM presentan altos índices de pobreza dentro de la Región Metropolitana de Santiago ${ }^{20}$. El hecho de que el AM reciba 
una jubilación le permite costear por ejemplo al menos las pilas que utiliza el audífono para su funcionamiento así como eventuales reparaciones 0 mantención.

Información como el grado de alfabetización del usuario (analfabeto, lee y escribe con dificultad y lee y escribe sin dificultad) se relaciona con las decisiones sobre la forma en que se efectuarán las instrucciones a ese paciente en particular, pero también permite al equipo tratante plantearse a futuro los mecanismos y estrategias comunicacionales que se tendrán que desarrollar para lograr una comunicación efectiva con la población atendida. El material educativo escrito que se utiliza para trabajar aspectos sobre el uso del aparato (cambio de pilas, limpieza, manejo del volumen) debía adecuarse a los niveles de analfabetismo que pudieran existir en este tipo de población; no obstante el analfabetismo es de $7 \%$ valor más bajo de lo que se esperaba para esta población, incluso más bajo que el promedio de la Región Metropolitana de Santiago que es de 9,3\% para mayores de 60 años ${ }^{21}$.

El $81 \%$ de AM reconocen no tener ningún nivel de participación en actividades sociales (de iglesia, clubes sociales o de AM), provocando un mayor aislamiento del AM de su entorno y de su comunidad. Tan alta cifra invita a reflexionar sobre las estrategias de difusión de estas actividades a nivel comunal, la posibilidad de acceso 0 la presencia de algún tipo de discapacidad como la auditiva que restringe al AM en su participación social debido a su problema de comunicación. En las comunas atendidas por el hospital existen 210 organizaciones sociales de AM (fuente SENAMA, Catastro nacional de organizaciones sociales de AM, 2005) por lo que la instancia existe. Este punto reviste una importancia muchas veces olvidada, la existencia de contactos sociales en los AM previene la aparición de alteraciones mentales, depresión, etc. Es así como un AM que vive cerca de sus hijos y participa activamente en actividades sociales presenta menos riesgo de presentar estas alteraciones $^{12}$.

Todos los datos recopilados son una primera aproximación a intentar conocer la realidad de nuestros adultos mayores. La motivación principal de realizar este estudio está relacionada con un cambio paradigmático actual que plantea una mirada más integral de los pacientes, en este caso de los adultos mayores; esta mirada tiene que integrar elementos de su contexto familiar y social, así como los factores de riesgo y factores protectores que nos hablan de un sujeto de atención que es protagonista de su proceso de integración.

Si pudiéramos, por ejemplo realizar una evaluación y contribuir de algún modo en el seguimiento (ejemplo, derivación al profesional correspondiente, etc.) más allá de lo técnico (tipo de pérdida auditiva, tipo de audífono, amplificación, etc.) podríamos postular algunas hipótesis diagnósticas sobre aspectos que pudieran modificarse previo 0 en forma posterior a la utilización del audífono, en parámetros como el de la participación con su comunidad, o realizar mecanismos de intervención que vayan en apoyo de los AM más aislados y limitados, donde el audífono por sí solo no es una ayuda suficientemente valorada cuando existen otras necesidades y limitaciones funcionales que impiden un buen aprovechamiento del beneficio recibido. En la medida que los profesionales de la salud comenzamos a trabajar con este grupo etáreo, comenzamos a observar más elementos que antes nos parecían invisibles; y es hora que comencemos a tomar en cuenta este conocimiento más integral de los AM, ya que la realidad apunta a que toda la problemática de la población envejecida será en corto plazo la directriz de nuestra acción como profesionales.

\section{CONCLUSIÓN}

Queda en evidencia la asociación de diversos factores no auditivos en los adultos mayores entrevistados. La asociación de uno o más de estos factores no auditivos pueden influir de manera importante en el desempeño comunicacional de un individuo al cual se implementa un sistema de asistencia como lo es un audífono. Por lo tanto se sugiere considerar estos factores al momento de comenzar con un proceso de rehabilitación auditiva en el adulto mayor.

Agradecimientos a la Dra. Margarita Arancibia S. por sus comentarios en la preparación de este trabajo. 


\section{BIBLIOGRAFÍA}

1. Gussekloo J. de Bont L E A, von Faber M. et al. Auditory rehabilitation of older people from the general population-the Leiden 85-plus Study. Br J Gen Pract 2003; 53(492): 536-40.

2. WeISTEIN BE. Behavionral Manifestations of AgeRelated Changes within the Ear. En: Geriatric Audiology. Thieme. 2000; 81-113.

3. Encuesta Nacional de Salud. Chile 2003. Biblioteca Virtual Ministerio de Salud. http:// epi.minsal.cl/epi/html/invest/ENS/ InformeFinalENS.pdf, consulta 24 Febrero 2009.

4. Manohar Bance. Hearing and Aging. CMaJ 2007; 176(7): 925-7.

5. Instituto Nacional de Estadísticas. Estadísticas sociales y culturales. www.ine.cl/canales/ chile_estadistico/estadisticas_sociales_culturales/ adultosmayores/pdf/cifrasmayores.pdf. Consulta 15 Septiembre 2008.

6. Marques A, Kozlowski L, Marques J. Auditory rehabilitation in elderly. Rev Bras Otorrinolaringol V70.n 6.809-17. Nov/dez 2004.

7. CHAO TK, CHEN TH. Cost-effectiveness of hearing aids in the hearing-impaired elderly: a probabilistic approach. Otol Neurotol 2008; 29(6): 776-83.

8. Vuorialho A, Karinen, P. Sorri M'Effect of hearing aids on hearing disability and quality of life in the elderly. Int J Audiol 2006; 45: 7, 400-5.

9. WeisteIn BE. Hearing Aids and Assistive Listening Devices. En: Geriatric Audiology. Thieme. 2000. Pags 209-264.

10. WeISTEIN BE. Audiologic Rehabilitation: An Integrated Approach. En: Geriatric Audiology. Thieme. 2000. Pags 171-207.

11. Kricos P. Audiologic Management of Older Adults With Hearing Loss and Compromised Cognitive/Psychoacoustic Auditory Processing Capabilities. Trends Amplif 2006; 10(1): 1-28. Review.
12. Tye-MurRay N. Foundations of Aural rehabilitation. Children, Adults, and their Family Member. $2^{a}$ edición. Thomson. Delmar Learning. 2004.

13. Gianopoulos I, Stephens D, Davis. A. Follow up of people fitted with hearing aids after adult hearing screening: the need for support after fitting. BMJ 2002; 325: 471.

14. López-Torres Hidalgo J, Gras CB, Lapeira JT, Verdejo MA, Del Campo JM, Rabadán FE. Functional status of elderly people with hearing Ioss. Arch Gerontol Geriatr 2008.

15. Vieira EP, Miranda EC, Calais LL, Carvalho LM, lóRIO MC, Borges AC. Group follow up proposal for elderly with hearing aids. Braz $J$ Otorhinolaryngol 2007; 73(6): 752-8.

16. Alberto J. Caban, David J. Lee, Orlando GómezMarín, Byron L. Lam, and D. Diane Zheng. Prevalence of Concurrent Hearing and Visual Impairment in US Adults: The National Health Interview Survey, 1997-2002. Am J Public Health 2005; 95(11): 1940-2.

17. ERBER NP. Use of hearing by older people: influence of non-auditory factors (vision, manual dexterity). Int J Audiol 2003; 42: 6, 21-25.

18. HUMES L, WILSON D, Humes A. Examination of differences between successful and unsuccessful elderly hearing aid candidates matched for age, hearing loss and gender. Int $J$ Audiol 2003; 42: 432-41.

19. Pichora-Fuller K. Cognitive aging and auditory information processing. Int J Audiol 2003; 42: 6, 26-32.

20. Encuesta de Caracterización Socioeconómica Nacional Casen 2006 http://www.mideplan.cl/ final/categoria. php?secid=25\&catid=124. Consulta 15 Septiembre 2008

21. Instituto Nacional de estadísticas. Estadísticas sociales y culturales. Adultos Mayores. http:// www.ine.cl/canales/chile_estadistico/ estadisticas_sociales_culturales/adultosmayores/ adultos_mayores.php. Consulta 16 Septiembre 2008. 